\title{
IDENTIFYING OPTICAL EINSTEIN RINGS
}

\author{
S. J. WARREN \\ Imperial College of Science Technology and Medicine \\ Prince Consort Rd, London SW7 2BZ, United Kingdom \\ P. C. HEWETT AND G. F. LEWIS \\ Institute of Astronomy \\ Madingley Road, Cambridge CB3 OHA, United Kingdom \\ P. MØLLER \\ Space Telescope Science Institute \\ 3700 San Martin Drive, Baltimore MD 21218, USA \\ A. IOVINO \\ Osservatorio Astronomico di Brera \\ Via Brera 28, I-20121 Milano, Italy \\ AND \\ P. A. SHAVER \\ European Southern Observatory \\ Karl-Schwarzschild-Strasse 2 \\ D-85748 Garching bei München, Germany
}

\section{Introduction}

The discovery of gravitationally lensed radio-rings (Hewitt et al. 1988) opened up a new line of attack on the problem of dark matter in galaxies. High-resolution radio observations (Langston et al. 1989) resolve structure tangentially and radially within the rings, providing sophisticated analysis routines (Kochanek \& Narayan 1992, Wallington, Narayan \& Kochanek 1994) with enough constraints to compute realistic models of the mass distribution within the deflectors (Kochanek 1995). Five such systems are now known and extensive programs to identify further examples are underway.

Exploiting the full potential of radio-rings is hampered by two factors. Firstly, a familiar problem within the gravitational lensing field, examples of the phenomenon are rare and while further systems will be identified the 
observational resources required are substantial. Secondly, once an example has been found it is in general very difficult to obtain unambiguous identifications of both the source and the deflector, since the optical counterparts of one or both can be extremely faint. Thus, of the five radio rings known, for only one, MG1654+134, are the redshifts of the source and deflector established. Without confirmed redshifts the basic geometry of the lens system remains unknown, severely limiting the quantitative information that can be deduced concerning the mass distribution within the deflector.

Miralda-Escudé and Lehár (1992) pointed out that the surface density of optical rings should greatly exceed that of radio rings. The deflector population, massive galaxies, is the same but the source counts of highredshift, intrinsically faint, marginally extended galaxies in the optical far exceeds the number of extended radio sources. The availability of samples of optical rings would provide complementary information to that obtained from studies of the radio rings. Working at optical wavelengths holds the promise of identifying a much larger number of systems, allowing powerful statistical analyses to be undertaken. It is also more likely that the redshifts of lens and deflector will be obtained as part of the discovery process. All the same, the dynamic range and resolution of modern radio arrays, (e.g. the VLA and MERLIN) operating at high radio frequencies, provide far more information for an individual radio ring, although the availability of the Hubble Space Telescope for imaging means the situation in the optical is much improved.

\section{THE OPTIMAL DEFLECTOR POPULATION}

The key to establishing an efficient strategy for identifying optical rings lies in targeting the fraction of the galaxy population most effective at producing strong gravitational lensing distortions of the numerous, distant, $z>1$, background source population. Confining attention to galaxies with the largest central velocity dispersions, $\sigma_{c}$, i.e. those with luminous massive bulges, maximizes the individual cross-sections, $\propto \sigma_{c}^{4}$, for strong lensing. A large surface density of lenses is also required to increase the total crosssection, effectively precluding the use of samples of nearby galaxies. The population of massive early-type galaxies in the redshift shell $z=0.3-0.5$ satisfies both requirements while also providing desirable geometries for lensing, with values of $D_{L S} / D_{O S} \sim 0.5$ for sources with redshifts $z>1.5$ $\left(D_{L S}\right.$ and $D_{O S}$ are the angular diameter distances between the lens and source, and the observer and source respectively). The angular radius of the Einstein ring of a galaxy of redshift $z=0.3$, modeled as a singular isothermal sphere with central velocity dispersion $\sigma_{c}=250 \mathrm{~km} \mathrm{~s}^{-1}$, for sources with redshift $z>1.5$, is $\sim 1.25$ arcsec. Rings with diameters $\sim 2.5 \operatorname{arcsec}$ 


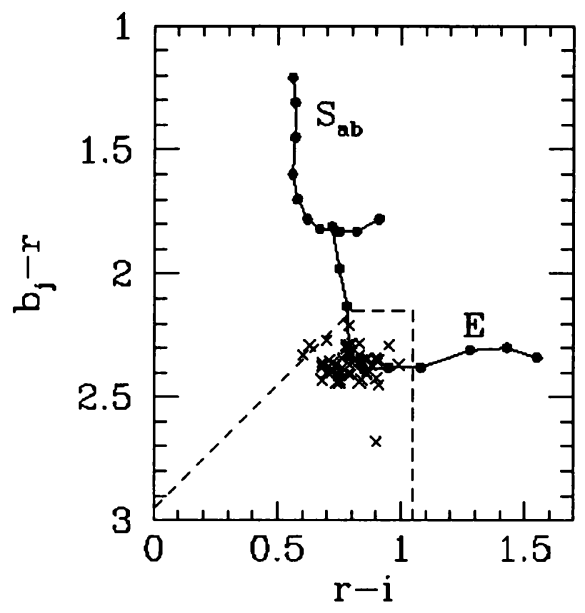

Figure 1. Two-color diagram, $\left(b_{j}-r, r-i\right)$, illustrating the predicted color-tracks for galaxies of type $\mathrm{Sab}$ and $\mathrm{E}$ over the redshift range $0.2<z<0.65$. The top of each locus corresponds to redshift $z=0.2$. Symbols along each locus indicate the redshift at intervals of $\Delta z=0.05$. The dashed line represents the color selection criteria for the galaxy sample, derived from APM scans of UK Schmidt plates, that forms the basis of our survey for optical rings. The crosses represent CCD photometry of 53 galaxies in the survey sample.

can be resolved from ground-based observatories in conditions of good atmospheric seeing and there is no reason in principle why they should not be detectable.

Early-type galaxies at distances of cosmological interest have been detectable for many years (Hamilton 1985). The relative ease with which such systems can be identified is due to the very strong spectral discontinuity arising from absorption line blanketing at $\sim 4000 \AA$, which, for nearby galaxies, produces large $U-B$ colors. The spectroscopic properties (determined from intermediate resolution observations of moderate signal-to-noise ratio) of the brightest early-type galaxies do not appear to evolve significantly out to redshifts $z>0.5$. Thus, as the redshift of the galaxy increases the $4000 \AA$ spectral discontinuity shifts to longer wavelengths, resulting in the colors $B-V, V-R, \ldots$ attaining very large values within specific redshift ranges. This evolutionary behavior is in marked contrast to the blueward 


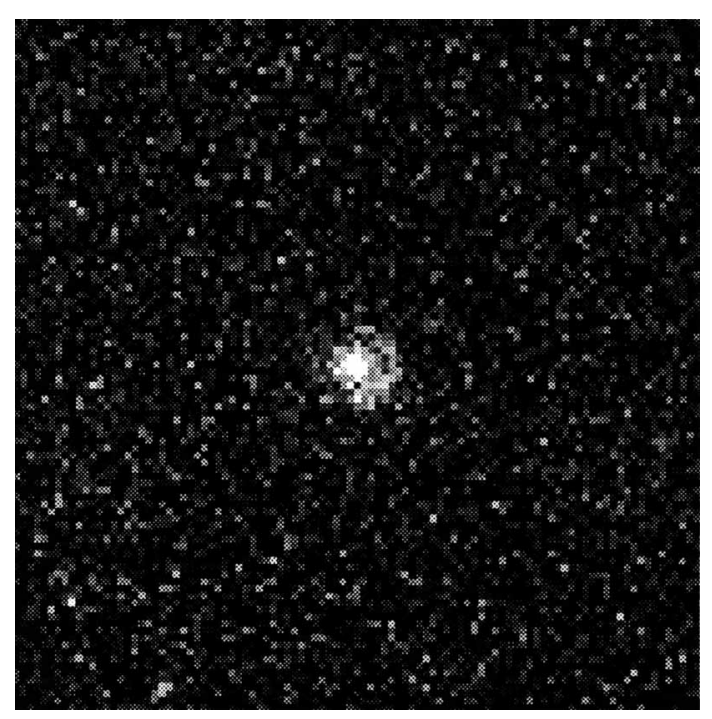

Figure 2. $3600 \mathrm{~s}$ narrow-band image of the optical ring, obtained at the NTT during 1994 December, in 0.9 arcsec seeing, using a $60 \AA$ wide filter centered at the wavelength of the emission line. The image is $26 \times 26$ arcsec in extent, with a pixel size of 0.26 arcsec. North is up and East to the left.

trend in color exhibited by the bulk of the galaxy population,

Much attention has naturally focussed on the properties of the strongly evolving galaxy population and the physical processes responsible for the observed behavior. The far less dramatic behavior of luminous early-type galaxies has not featured as prominently. The reasons for this are twofold; (a) the selection of galaxy samples using blue passbands - an $L^{*}$ early-type galaxy at $z=0.4$ has a $B$ magnitude $m_{B} \sim 23$, and only the faintest spectroscopic surveys achieve the necessary depth, and (b) the low surface density on the sky - in the redshift shell $0.3<z<0.5$ only tens of objects per square degree are expected, and existing spectroscopic surveys contain very few examples. However, the use of wide-field photographic plate material in the $R$ and $I$ passbands enables a large sample of distant early-type galaxies to be defined.

\section{THE FIRST CANDIDATE EINSTEIN RING}

Our investigation employs APM scans of UK Schmidt Telescope (UKST) plates in $B_{J}, R$ and $I$ to identify the population of luminous early-type galaxies within the redshift shell $z=0.3-0.5$. Galaxies are selected to lie in the color range $r-i<1.05, b_{j}-i>2.95$, and $b_{j}-r>2.15$ (Figure 1 ), with magnitudes $16.4<m_{i}<18.85$. A galaxy at redshift $z=0.4$ ap- 
pearing at the sample limit has absolute magnitude $M_{B} \sim-21.5\left(q_{0}=0.5\right.$, $H_{0}=50 \mathrm{kms}^{-1} \mathrm{Mpc}^{-1}, \Lambda=0.0$ ). The survey to date covers 5 UKST fields, $150 \mathrm{deg}^{2}$, and the surface density of candidates is $\sim 40 \mathrm{deg}^{-2}$. Spectroscopy of $162 \mathrm{objects}$ within an area of $3 \mathrm{deg}^{2}$ demonstrates the effectiveness of the selection procedures, producing 154 confirmed galaxies, only two of which have $z<0.3$, and only $8 \mathrm{M}$-stars, a contamination rate of $<5 \%$.

Among the apparently normal galaxy spectra one object, a luminous early-type galaxy at redshift $z=0.485$, possesses a strong emission feature at $5588 \AA$. No other emission lines are evident over the wavelength range $\lambda \lambda 4456-7912 \AA$. Narrow-band imaging of the system at the emission-line wavelength reveals a semi-circular arc, radius $r=1.35$ arcsec, centered on the peak of the galaxy surface-brightness distribution (Figure 2). The properties of the system can be reproduced by a gravitational lens model in which the deflector has a constant mass-to-light ratio (corrected for evolution) of $\mathrm{M} / \mathrm{L}_{\mathrm{B}(0)} \sim 10\left(H_{0}=50\right)$ and the source is an intrinsically small but resolved (FWHM $\sim 0.2$ arcsec) object at high redshift, either at $z=3.597$ (if the line is $\operatorname{Ly} \alpha \lambda 1216$ ), or at $z=2.607$ (if the line is CIV $\lambda 1549$ ). Modeling of the system suggests an amplification of a factor $\sim 20$ with an unlensed magnitude for the source of $m_{V} \sim 27$.

Alternatives to the lensing explanation, in which the emission feature is associated with [OII] $\lambda 3727$, with a velocity difference of $2900 \mathrm{~km} \mathrm{~s}^{-1}$ relative to the absorption-line redshift of the galaxy, appear to be extremely improbable. The system is almost certainly the first Einstein ring to be discovered in the optical, although unambiguous confirmation requires the detection of a second emission line. A full description of the observations and modeling is given by Warren et al. (1996).

\section{FUTURE WORK}

In common with most other examples of gravitational lensing phenomena, obtaining quantitative astrophysical information of interest on both the deflectors and the sources depends on the ability to identify a significant number of similar systems in a well-defined and efficient fashion. The multicolor selection technique described in $\S 2$ enables us to target distant, massive, early-type galaxies with relative ease. The availability of widefield spectroscopic systems, particularly the $2 \mathrm{dF}$ on the Anglo-Australian Telescope means thousands of such galaxies can be identified with only a modest investment of time - the $2 \mathrm{dF}$ is capable of obtaining spectra of $\sim 120$ of our target galaxies in a single pointing of a few hours.

The discovery of further optical rings is predicated on the existence of an emission line(s) in the sources, thus producing a strong but readily quantifiable selection criterion, and, in general, allowing the measurement of the 


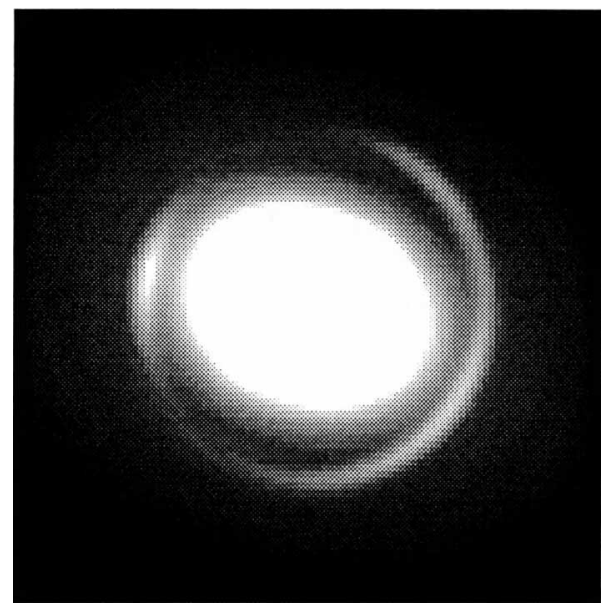

Figure 3. Simulation of 16 orbit observation with the HST PC of the optical ring. The simulation is based on the simple model for the system in which mass traces light in the deflecting galaxy, $z=0.485$, and the source, $z=3.597$, has a Gaussian profile with a FWHM= 0.2 arcsec. The image shows the field after subtraction of the galaxy. Contributions to the noise from the detector (read-out noise and dark current), and Poisson noise from the deflecting galaxy, the source, and from sky, are included. North is up and East to the left.

redshift of the source. Application of the statistical techniques developed by Kochanek (1993a, 1993b) to a sample of $10-20$ lenses, coupled with high-resolution imaging from HST of individual systems (Figure 3), would produce unique information concerning the surface density and properties of emission-line objects in the crucial redshift range $z=2-4$ at magnitudes as faint as $m_{V} \sim 27$. Equally important, such a sample will provide quantitative constraints on the distribution of dark matter associated with early-type galaxies.

\section{References}

Hamilton, D., 1985, ApJ, 297, 371

Hewitt, J.N., Turner, E.L., Schneider, D.P., Burke, B.F., Langston, G.I. \& Lawrence, C.R., 1988, Nature, 333, 537

Kochanek, C.S., 1993a, ApJ, 417, 438

Kochanek, C.S., 1993b, ApJ, 419, 12

Kochanek, C.S., 1995, ApJ, 445, 559

Kochanek, C.S. \& Narayan, R., 1992, ApJ, 401, 461

Langston, G.I., et al., 1989, AJ, 97, 1283

Miralda-Escudé, J. \& Lehár, J., 1992, MNRAS, 259, 31P

Wallington, S., Narayan, R. \& Kochanek, C.S., 1994, ApJ, 426, 60

Warren, S.J., Hewett, P.C., Lewis, G.F., Møller, P., Iovino, A. \& Shaver, P.A., 1996, MNRAS, in press 